\title{
Association of Depressive Symptoms with Iron Management in Patients on Maintenance Hemodialysis: A Cross-sectional Study
}

\author{
Yoshihiro Tsuji ${ }^{1, ~}$, Naoki Suzuki ${ }^{2}$, Yasumasa Hitomi ${ }^{2}$, Yuko Mizuno-Matsumoto ${ }^{3}$, Toshiko Tokoro ${ }^{4}$, \\ Masato Nishimura ${ }^{5}$ \\ ${ }^{1}$ Department of Medical Engineering, Faculty of Health Sciences, Morinomiya University of Medical Sciences, Osaka, Japan \\ ${ }^{2}$ Department of Clinical Engineering, Tojinkai Hospital, Kyoto, Japan \\ ${ }^{3}$ Graduate School of Applied Informatics, University of Hyogo, Kobe, Japan \\ ${ }^{4}$ Department of Nephrology, Tojinkai Satellite Clinic, Kyoto, Japan \\ ${ }^{5}$ Cardiovascular Division, Tojinkai Satellite Clinic, Kyoto, Japan
}

Email address:

yoshihiro_tsuji@morinomiya-u.ac.jp (Y. Tsuji)

${ }^{*}$ Corresponding author

\section{To cite this article:}

Yoshihiro Tsuji, Naoki Suzuki, Yasumasa Hitomi, Yuko Mizuno-Matsumoto, Toshiko Tokoro, Masato Nishimura. Association of Depressive Symptoms with Iron Management in Patients on Maintenance Hemodialysis: A Cross-sectional Study. International Journal of Biomedical Engineering and Clinical Science. Vol. 6, No. 2, 2020, pp. 46-54. doi: 10.11648/j.ijbecs.20200602.14

Received: May 5, 2020; Accepted: June 15, 2020; Published: June 29, 2020

\begin{abstract}
Background: Iron status has a critical role in depressive symptoms, but evaluation of depressive symptoms associated with iron metabolism is not usually included as a clinical parameter in hemodialysis (HD) patients. We aimed to assess the correlations between depressive symptoms and clinical, demographic, and laboratory variables including iron metabolism. Methods: One hundred and thirty-eight HD patients were evaluated in this study. The Beck Depression Inventory Second Edition (BDI-II) was used to quantify levels of depressive symptoms. BDI-II scores $\geq 14$ were defined as depressive symptoms. Mean age, duration of HD, haemoglobin levels, serum ferritin levels, serum iron levels, transferrin saturation (TSAT), total iron binding capacity, serum albumin levels, and C-reactive protein were included in the model. Patients were categorized into four groups according to serum ferritin levels and TSAT. Backward stepwise logistic regression analysis was performed and odds ratios (ORs) and $95 \%$ confidence intervals derived. Results: Depressive symptoms were significantly associated with increased serum ferritin levels (OR, 1.010; $\mathrm{p}=0.0008$ ). Compared with group 1 (ferritin $<100 \mathrm{ng} / \mathrm{dL}$, TSAT $\geq 20 \%$ ) as reference, ORs for depressive symptoms were significantly increased in group 4 (ferritin $\geq 100 \mathrm{ng} / \mathrm{dL}$, TSAT $<20 \%)(\mathrm{OR}, 6.419 ; \mathrm{p}=0.0073)$. Conclusion: Higher serum ferritin levels and decreased iron utilization efficiency were found to be involved in depressive symptoms among patients undergoing HD. Understanding the pathophysiology of depressive symptoms could provide insights into the design of clinical iron management in HD patients.
\end{abstract}

Keywords: Depressive Symptoms, Hemodialysis, Iron Management

\section{Introduction}

Depressive symptoms represent the main psychological disorder in patients undergoing maintenance hemodialysis (HD) $[1,2]$ and are associated with increased mortality in patients undergoing HD [3, 4]. Moreover, depressive symptoms conventionally occur in patients who have just begun HD treatment; depression generally tends to resolve in the maintenance phase. Despite the long-term survival benefit to patients resulting from advances in medical technology, depressive symptoms can still be present. In recent years, mental or psychological care has become a focus of dialysis therapy, to minimize systemic complications that appear together with the long-term reduction in quality of life.

Numerous studies have addressed that iron status has a critical role in brain function and cerebral mechanisms [5-7]. 
Previous studies have pointed out that depression is associated with decreased serum ferritin concentrations $[8,9]$. Some studies have reported that there was no significant association between depression and serum ferritin concentration $[10,11]$. Iron is an important component in the treatment of anaemia among HD patients. Serum ferritin levels and transferrin saturation (TSAT) are used to assess iron status, as a guide for anaemia therapy; both iron replacement and iron maintenance therapy are required to treat anaemia. However, not all previous studies have confirmed a relationship between depressive symptoms and iron status, and little is known about how to manage iron in HD patients from the standpoint of depressive symptoms. In this study, we aimed to assess the relationship among depressive symptoms and clinical, demographic, and laboratory variables including iron metabolism. Our present findings showed an association between depressive symptoms and iron status in HD patients.

\section{Methods}

\subsection{Ethics Approval and Consent to Participate}

This study was approved by The Ethics Committee for Human Research of Tojinkai Hospital. As collected data were obtained from routine clinical practice further consents were waived. All procedures performed in studies involving human participants were in accordance with the ethical standards of the institutional, and national research committee at which the studies were conducted (IRB approval number NCT03096626) and with the 1964 Helsinki declaration and its later amendments or comparable ethical standards.

\subsection{Study Population}

The One hundred and fifty-two patients (mean age, $65.9 \pm$ 11.4 years; women, $50 \%$; mean duration of $\mathrm{HD}, 14.2 \pm 8.8$ years) undergoing maintenance HD three times weekly for over 1 year were included in this cross-sectional study. Data were obtained from the Tojinkai hospital. Subjects in the present study were sampled by a random process. Patients with chronic hepatitis B or C were excluded, as were 14 patients who refused to enrol in the present study and those who could not complete the questionnaire by themselves. Patients with low cognitive function and who declined to be enrolled in the study and those who could not complete the questionnaire themselves were excluded. As a result, 138 patients were enrolled as study participants (mean age, $66.2 \pm$ 11.1 years; women $51 \%$; mean duration of HD, $14.3 \pm 8.7$ years). We extracted the following clinical characteristics from patients' medical records: sex, mean age, height, dry weight (DW), body mass index (BMI), duration of HD, HD time, blood flow rate during $\mathrm{HD}$, presence of underlying diabetes mellitus (DM), and $\mathrm{Kt} / \mathrm{V}_{\text {urea }}$. Calculations were made of erythropoiesis-stimulating agents (ESA) and intravenous iron preparations in average monthly doses for the past 3 months. The ESA was expressed as dosage of darbepoetin alfa $(\mu \mathrm{g})$. Blood samples $(4 \mathrm{~mL})$ were obtained just before initiation of HD at the beginning of a regular HD session on the first day of the week. Laboratory data including haemoglobin levels, haematocrit, serum ferritin levels, serum iron levels, total iron binding capacity (TIBC), blood urea nitrogen (BUN), serum albumin levels, creatinine $(\mathrm{Cr})$, and C-reactive protein (CRP) were obtained. Serum ferritin levels and CRP were measured using the latex agglutination turbidimetry method. Serum iron levels were measured with the Nitroso-PSAP method. TSAT was calculated as serum iron levels divided by TIBC. The geriatric nutritional risk index (GNRI) was calculated as follows: GNRI $=[14.89 \times$ serum albumin levels $(\mathrm{g} / \mathrm{dL})+41.7 \times$ (body weight/ideal body weight)] [12]. Ideal body weight was calculated using the patients' height and a BMI of $22[13,14]$.

\subsection{Assessment of Depressive Symptoms}

The Beck Depression Inventory Second Edition (BDI-II, Japanese version) was used to evaluate patients, so as to quantify depression levels [15]. The BDI-II has been the most widely used instrument for self-assessment of depression among patients undergoing dialysis [16, 17]. There are 21 items on the self-administered questionnaire assessing both the presence and severity of depression, with four possible responses to statements that are scored from 0 to 3 and total scores ranging from 0 to 63 [18]. A higher BDI-II score indicates a greater level of depression.

\subsection{Statistical Analysis}

In the present study, we defined patients with BDI-II scores $\geq 14$ as having depressive symptoms [19]. Patients were divided into two groups according to their BDI-II score, a non-depressed group (BDI-II score $<14$ ) and depressed group (BDI-II score $\geq 14$ ). The clinical characteristics of the two groups were compared using the Mann-Whitney $U$ test for mean age, height, DW, BMI, duration of HD, HD time, blood flow rate during $\mathrm{HD}$, and laboratory variables. Because of nonnormality distribution of some clinical variables by using the Shapiro-Wilk normality test, nonparametric test was performed as a distribution-free test. The Fisher's exact probability test was used for sex and the presence of underlying DM. Logistic regression analysis was performed to select determinants between patients grouped by the presence or absence of depressive symptoms according to BDI-II score, the dependent variable. Mean age, duration of HD, haemoglobin levels, serum ferritin levels, serum iron levels, TSAT, TIBC, serum albumin levels, and CRP were included in the model. Data are presented as odds ratios (ORs) with $95 \%$ confidence intervals (CIs). Patients were categorized into four groups according to their serum ferritin levels and TSAT. Here, we used cut-off values for serum ferritin levels and TSAT of $100 \mathrm{ng} / \mathrm{mL}$ and $20 \%$, respectively. Japanese guidelines for renal anaemia in chronic kidney disease recommend administration of iron to patients with serum ferritin levels $<$ $100 \mathrm{ng} / \mathrm{mL}$ and with TSAT $<20 \%$ [20]. These are the values that the guidelines of 2008 Japanese Society for Dialysis Therapy for treating anemia in CKD recommend that iron be 
administered to patients [20]. The four patient groups were as follows: Group 1, serum ferritin levels $<100 \mathrm{ng} / \mathrm{dL}$, TSAT $\geq 20 \%$; group 2 , serum ferritin levels $\geq 100 \mathrm{ng} / \mathrm{dL}$, TSAT $\geq 20 \%$; group 3, serum ferritin levels $<100 \mathrm{ng} / \mathrm{dL}$, TSAT $<20 \%$; and group 4 , serum ferritin levels $\geq 100 \mathrm{ng} / \mathrm{dL}$, TSAT $<20 \%$. For comparisons among the groups, a backward stepwise logistic regression analysis test was performed to identify those risk factors that contribute to depressive symptoms. All statistical analyses were performed using of JMP 10 software (SAS Institute, Inc., Cary, NC, USA), with a $p$-value $<0.05$ considered statistically significant.

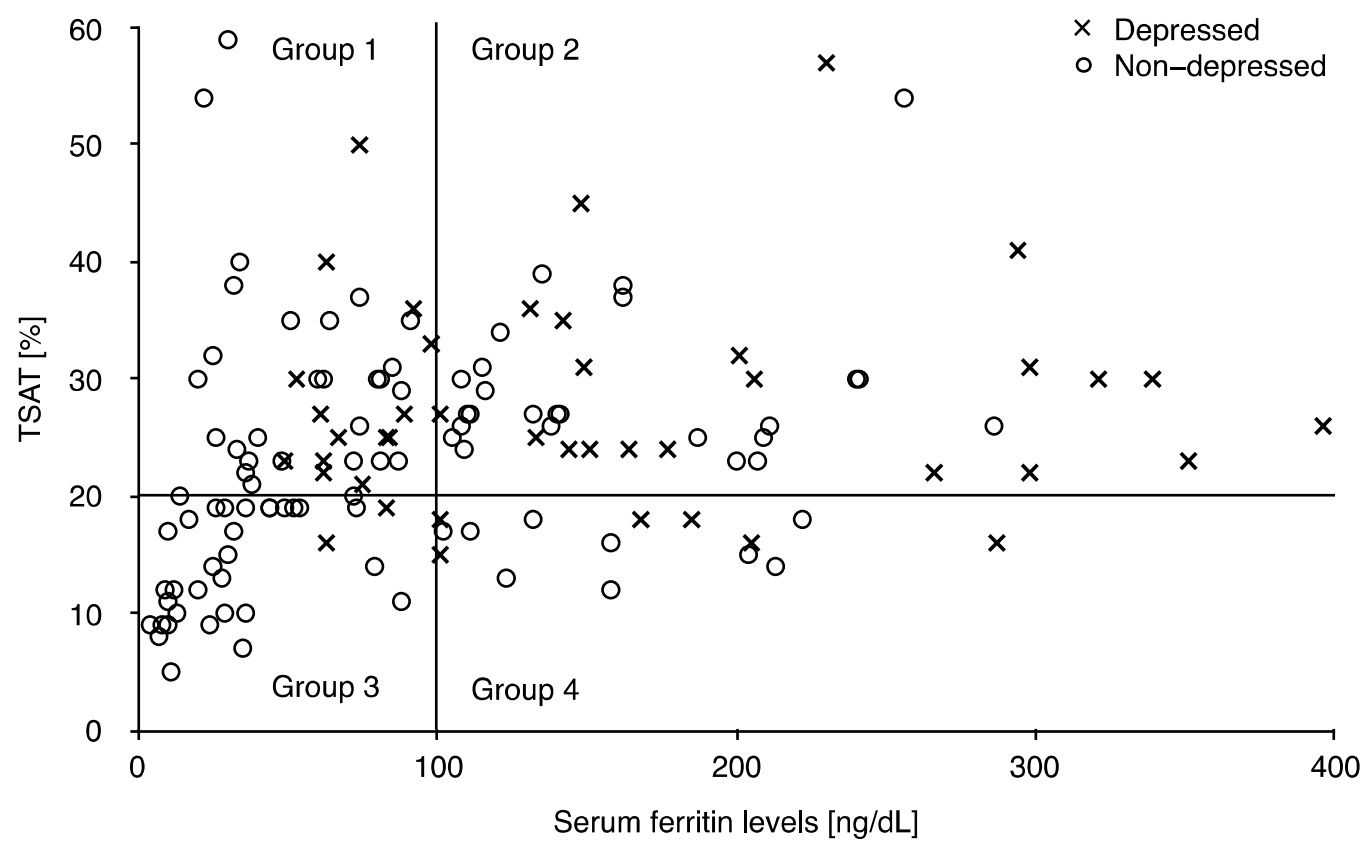

Figure 1. Distribution of depression symptoms according to serum ferritin levels and TSAT (transferrin saturation) $\times$ and $\bigcirc$ indicate patients with depression and no depression symptoms, respectively. Patients were categorized into four groups according to serum ferritin levels and TSAT as follows: Group 1 ( $n=43$ ), TSAT $\geq 20 \%$ and ferritin $<100 \mathrm{ng} / \mathrm{dL} ;$ Group 2 ( $n=47)$, TSAT $\geq 20 \%$ and ferritin $\geq 100 \mathrm{ng} / \mathrm{dL} ;$ Group 3 ( $n=33$ ), TSAT $<20 \%$ and ferritin $<100 \mathrm{ng} / \mathrm{dL} ;$ Group 4 $(n=15), T S A T<20 \%$ and ferritin $\geq 100 \mathrm{ng} / \mathrm{dL}$.

\section{Results}

\subsection{Demographic Characteristics)}

Table 1 shows clinical characteristics categorized by the presence of depressive symptoms according to scores on the BDI-II. Among all patients, 43 with a BDI-II score $\geq 14$ were considered depressed (31\%); 95 patients with BDI-II score $<$ 14 were considered non-depressed (69\%). The median (interquartile range: IQR) values of BDI-II score were 20 (22.0-18.0) in the depressed group and 6.0 (8.5-3.0) in the non-depressed group. The demographic, clinical, and laboratory characteristics of all patients are summarized in Table 1. Upon comparing the depressed and non-depressed groups, BDI-II scores, serum ferritin levels, and TSAT were found to be significantly higher among participants in the depressed group than those among the non-depressed group ( $p$ $<0.0001, p<0.0001, p=0.0151$, respectively). TIBC was significantly lower in the depressed group than in the non-depressed group $(p=0.0026)$.

\subsection{Relationships Between Depressive Symptoms and Clinical Variables}

Table 2 shows the results of logistic regression analysis of clinical variables. Relative to the non-depressed group, the depressed group had increased ORs for serum ferritin levels (OR, 1.010; 95\% CI: 1.004-1.016; $p=0.0008$ ). No significant differences were found between the depressed and non-depressed group for other clinical variable outcomes.

\subsection{Relationships Between Serum Ferritin Levels and TSAT}

Figure 1 shows the distribution of patients with depressive symptoms and no depressive symptoms according to serum ferritin levels and TSAT. Table 3 shows the results of logistic regression analysis with ORs and 95\% CIs for group 1 compared with the other three groups. When comparing group 1 (serum ferritin levels $<100 \mathrm{ng} / \mathrm{dL}$, TSAT $\geq 20 \%$ ) with group 2 (serum ferritin levels $\geq 100 \mathrm{ng} / \mathrm{dL}$, TSAT $\geq 20 \%$ ), group 3 (serum ferritin levels $<100 \mathrm{ng} / \mathrm{dL}$, TSAT $<20 \%$ ) and group 4 (serum ferritin levels $\geq 100 \mathrm{ng} / \mathrm{dL}$, TSAT $<20 \%$ ), ORs for depressive symptoms were significantly higher in group 4 (OR, 6.419; 95\% CI, 1.583-43.523; $p=0.0073$ ). In a comparison of group 1 with groups 2 and 3, the differences were not statistically significant $(p=0.0868, p=0.5333$, respectively.

\section{Discussion}

To identify the risk factors for depressive symptoms in HD patients, we focused on assessing those factors responsible for 
depression including clinical, demographic, and laboratory variables that have been previously suggested. In our study, BDI-II score was used to assess the level of depressive symptoms, and the prevalence of depressive symptoms was $32.2 \%$ among our patients. In this study, the primary finding was that a higher serum ferritin level was an independent risk factor for depressive symptoms. Secondary findings were that higher serum ferritin levels and lower TSAT were associated with depressive symptoms. That is, ORs for patients with depressive symptoms were significantly higher among patients who had serum ferritin levels $\geq 100 \mathrm{ng} / \mathrm{dL}$ and TSAT $<20 \%$ than in patients with serum ferritin levels $<100 \mathrm{ng} / \mathrm{dL}$ and $\mathrm{TSAT} \geq 20 \%$.
Iron is an essential micronutrient for all living organisms as it is required for adequate erythropoietic function, oxidative metabolism, and cellular immune responses. However, excess iron causes various disorders. In general, iron promotes the production of free radical ions and damages cells or tissues, so iron is fundamentally a harmful substance to humans [21-23]. Most patients undergoing maintenance $\mathrm{HD}$ receive a combination of ESA and intravenous iron therapy for anaemia management. Japanese Guidelines for Renal Anemia in Chronic Kidney Disease recommend a target haemoglobin level in HD patients of $11-12 \mathrm{~g} / \mathrm{dL}$, and administration of iron therapy for patients with TSAT $<20 \%$ and serum ferritin levels $<100 \mathrm{ng} / \mathrm{mL}$ [20].

Table 1. Clinical characteristics and prevalence of depression according to the Beck Depression Inventory among HD patients.

\begin{tabular}{llll}
\hline Clinical variable & Depressed (n=43) & No-Depressed (n=95) & p-value \\
\hline BDI score (IQR) & $20(22.0-18.0)$ & $6.0(8.5-3.0)$ & $<0.00011^{* *}$ \\
Men / Women & $20 / 23$ & $48 / 47$ & 0.7152 \\
Mean age, yr (SD) & $65.7(11.3)$ & $66.4(11.1)$ & 0.6640 \\
Height, cm (SD) & $161.3(9.9)$ & $161.2(9.1)$ & 0.7077 \\
Dry weight, kg (SD) & $56.3(11.5)$ & $57.3(12.7)$ & 0.9519 \\
Body mass index, kg/m (SD) & $21.5(3.6)$ & $21.7(4.3)$ & 0.7250 \\
Duration of HD, yr (SD) & $14.3(9.4)$ & $14.3(8.4)$ & 0.7130 \\
HD time, hr (SD) & $4.5(0.5)$ & $4.7(0.6)$ & 0.0716 \\
Blood flow during dialysis, ml/min (SD) & $287.2(23.8)$ & $289.1(27.2)$ & 0.6450 \\
DM / non-DM & $14 / 29$ & $19 / 76$ & 0.0722 \\
Hemoglobin levels, g/dL (IQR) & $10.9(11.5-10.4)$ & $11.0(11.7-10.3)$ & 0.5098 \\
Hematocrit,\% (IQR) & $33.0(34.3-31.7)$ & $34.1(36.0-31.7)$ & 0.1252 \\
Serum ferritin levels, ng/dL (IQR) & $142.0(205.5-83.0)$ & $72.0(119.8-30.0)$ & $<0.0001 * *$ \\
Serum iron levels, $\mu \mathrm{g} / \mathrm{dL}$ (IQR) & $65.0(80.5-53.0)$ & $58.0(75.0-42.0)$ & 0.0820 \\
TSAT,\% (IQR) & $25.0(31.0-22.0)$ & $23.0(29.8-16.3)$ & $0.0151 *$ \\
TIBC, $\mu \mathrm{g} / \mathrm{dL}$ (IQR) & $243.0(265.5-228.0)$ & $270.0(305.8-236.3)$ & $0.0026 * *$ \\
Serum albumin levels, g/dL (IQR) & $3.9(4.1-3.7)$ & $3.9(4.1-3.7)$ & 0.7453 \\
CRP, mg/dL (IQR) & $0.084(0.308-0.036)$ & $0.092(0.200-0.048)$ & 0.7560 \\
Serum creatinine levels, g/dL (IQR) & $10.2(11.9-9.4)$ & $11.0(12.0-9.9)$ & 0.3476 \\
Blood urea nitrogen, mg/dL (IQR) & $59.0(68.5-49.5)$ & $60.0(69.0-52.3)$ & 0.5792 \\
ESAs, $\mu$ g/week (SD) & $12.1(10.4)$ & $13.0(17.9)$ & 0.8428 \\
Intravenous iron preparation, mg (SD) & $8.4(9.7)$ & $7.6(9.7)$ & 0.6873 \\
GNRI (SD) & $96.1(5.3)$ & $96.9(5.0)$ & 0.4663 \\
Kt/ wrea $(S D)$ & $1.95(0.32)$ & $1.96(0.30)$ & 0.7458 \\
\hline
\end{tabular}

Abbreviations: BDI, Beck Depression Inventory; HD, hemodialysis; DM, diabetes mellitus; TAST, transferrin saturation; TIBC, total iron binding capacity; CRP, C-reactive protein; ESA, erythropoiesis-stimulating agent; GNRI, geriatric nutritional risk index.

Notes: Results reported as mean with standard deviation (SD) or median changes with interquartile range (IQR). Patients were divided into two groups according to BDI scores: non-depressed group (BDI score $<14$ ), and depressed group (BDI score $\geq 14$ ). Differences between the two groups were assessed by Mann-Whitney $U$-test or Fisher's exact probability tests.

$* * p<0.01, * p<0.05$ for comparisons between the groups.

Table 2. Logistic regression analysis of the relationship between depression symptoms and clinical variable.

\begin{tabular}{lll}
\hline Clinical variable & Odds ratio (95\% CI) & $p$-value \\
\hline Mean age, yr & $0.984(0.941-1.029)$ & 0.4758 \\
Duration of HD, yr & $0.992(0.942-1.043)$ & 0.7428 \\
Hemoglobin levels, g/dL & $1.034(0.616-1.712)$ & 0.8971 \\
Serum ferritin levels, ng/dL & $1.010(1.004-1.016)$ & $0.0008 * *$ \\
TSAT, $\%$ & $1.031(0.983-1.083)$ & 0.2055 \\
TIBC, $\mu$ g/dL & $0.988(0.974-1.001)$ & 0.0846 \\
Serum albumin levels, g/dL & $1.867(0.257-13.805)$ & 0.5349 \\
CRP, mg/dL & $0.741(0.218-1.989)$ & 0.5797 \\
\hline
\end{tabular}

Abbreviations: HD, hemodialysis; TAST, transferrin saturation; TIBC, total iron binding capacity; CRP, C-reactive protein; CI, confidence interval. $* * p<0.01$. 
Table 3. Odds ratios for the presence of depression among haemodialysis patients in group 1 (ferritin $<100 \mathrm{ng} / \mathrm{dL}$, TSAT $\geq 20 \%$ ) compared with the other groups, by serum ferritin levels and TSAT.

\begin{tabular}{llll}
\hline & Number of patients (depressed) & Adjusted odds ratio (95\% CI) & $\boldsymbol{p}$-value \\
\hline Group 1 Serum ferritin levels $<100 \mathrm{ng} / \mathrm{dL}$ TSAT $\geq 20 \%$ & $43(14)$ & Reference & - \\
Group 2 Serum ferritin levels $\geq 100 \mathrm{ng} / \mathrm{dL}$ TSAT $\geq 20 \%$ & $47(21)$ & $0.460(0.087-0.184)$ & 0.0868 \\
Group 3 Serum ferritin levels $<100 \mathrm{ng} / \mathrm{dL}$ TSAT $<20 \%$ & $33(2)$ & $0.670(0.191-2.421)$ & 0.5333 \\
Group 4 Serum ferritin levels $\geq 100 \mathrm{ng} / \mathrm{dL}$ TSAT $<20 \%$ & $15(6)$ & $6.419(1.583-43.523)$ & $0.0073 *$ \\
\hline
\end{tabular}

Abbreviations: CI, confidence interval; TAST, transferrin saturation. Note: Group 1 is the reference. ${ }^{*} p<0.051$.

Haemodialysis patients are reported to have a high prevalence of inflammation that is associated with survival [24]. Patients with inflammation often develop anaemia that is associated with iron metabolism abnormalities caused by the expression of divalent metal transporter and transferrin receptors, which are iron uptake proteins, or a decrease in the expression of ferroportin 1, which is an iron export protein. As a result, iron localization, in which iron is enclosed within cells, causes the use of iron effective for haematopoiesis to decrease. That is, reticuloendothelial iron release disorder occurs, and iron is released from the mesenchymal system into the blood [25].

In this study, patients without depressive symptoms had serum ferritin levels $<100$ and TSAT $\geq 20 \%$, indicating that iron was used effectively to treat anaemia. That is, iron release from iron metabolic cells, including in the mesenchymal system, is thought to have occurred in a proper manner. On the other hand, in patients with depressive symptoms, TSAT was more than $25 \%$ but ferritin was higher and TIBC was lower than in patients with no depressive symptoms. These results suggest that patients with depressive symptoms might be in a state of functional iron deficiency. Functional iron deficiency is defined as a state in which there is insufficient incorporation of iron into erythroid precursors in the face of adequate body iron stores [26]. Additionally, higher serum ferritin levels and lower TSAT were associated with depressive symptoms. That is, ORs for patients with depressive symptoms were significantly higher in patients with serum ferritin levels $\geq 100$ $\mathrm{ng} / \mathrm{dL}$ and TSAT $<20 \%$ than those with serum ferritin levels $<100 \mathrm{ng} / \mathrm{dL}$ and TSAT $\geq 20 \%$. Classification by serum ferritin levels and TSAT values could be thought of as follows. Group 1 (serum ferritin levels $<100 \mathrm{ng} / \mathrm{dL}$ and TSAT $\geq 20 \%$ ) represents a state in which there is sufficient iron incorporation into erythroid precursors during iron absorption and recycling; group 2 (serum ferritin levels $\geq 100 \mathrm{ng} / \mathrm{dL}$, TSAT $\geq 20 \%$ ), a state of iron overload; group 3 (serum ferritin levels $<100 \mathrm{ng} / \mathrm{dL}$, TSAT $<20 \%$ ), a state of iron deficiency; and group 4 (serum ferritin levels $\geq 100 \mathrm{ng} / \mathrm{dL}$, TSAT $<20 \%$ ), a state of functional iron deficiency.

In HD patients, iron is often supplemented intravenously because intravenous iron administration is effective in correcting anaemia. Excessive accumulation of iron is thought to cause tissue damage and to possibly contribute to arteriosclerosis [27-28]. Excessive accumulation of iron in regions of the brain that undergo degeneration is a cause of neurological diseases such as Alzheimer disease, Parkinson disease, and other disorders [29-34]. Furthermore, it has been reported that hippocampus volume and function are decreased in depressed patients. Although the precise role of iron in the cause of many neurodegenerative diseases is unclear, a previous study reported that neurodegeneration accompanied by iron accumulation disrupts the neural network in the associative field of hippocampus and cortex and gradually causes neurologic symptoms, such as dementia, to manifest [35]. The results of the present study suggest the appearance of depressive symptoms may be associated with neurodegeneration as a result of brain iron accumulation by an overload of iron that is not effectively used for haematopoiesis. Iron excretion disorder among HD patients occurs when excess free iron within cell causes cellular injury. Therefore, serum ferritin levels and TSAT must be simultaneously controlled and kept at levels $<100 \mathrm{ng} / \mathrm{dL}$ and $\geq 20 \%$, respectively. The involvement of iron in depressive symptoms needs further elucidation but understanding the pathophysiology of depressive symptoms could provide insight into the role of iron and help in the design of clinical iron management for HD patients.

There are some limitations in our study. The observed associations were obtained by using a cross-sectional design, which does not permit us to draw casual conclusions with confidence. Therefore, more prospective studies to determine whether depressive symptoms among HD patients are associated with iron status are needed. Depressive symptoms were estimated from using only the BDI-II; therefore, we could not eliminate the possibility of a multifaceted psychological impact on the score results. Using two or more different kinds of questionnaire for psychological testing is required to more thoroughly assess depressive symptoms. In addition, there is a need to conduct the psychiatric interviewer or depressive symptoms by a screening test or screening low cognitive function for the presence of clinical depression by a trained psychiatric interviewer. In this study, we did not consider the association between iron status and dietary habits; therefore, we cannot exclude the possibility that the observed associations had to do with dietary intake of iron. Iron status and dietary iron intake (e.g., iron-based phosphorus binders) should be considered in future investigation of associations between iron status and depressive symptoms among HD patients. Serum albumin levels and CRP, which are important indexes of biomarkers in HD patients, were not found to be associated with depressive symptoms in the present study. Several other studies have shown that lower serum albumin levels and higher CRP caused depressive symptoms [36-38]. However, further prospective trials may be required to examine the impact of nutritional and inflammation status upon depressive symptoms in HD patients and to determine whether serum albumin levels 
and higher CRP are associated with depressive symptoms. This observational retrospective study allowed only limited conclusions. We could not prove cause-and-effect relationship between iron metabolism and the presence of depressive symptoms. Since serum ferritin is both an iron storage protein and an acute phase reactant, further prospective studies are needed for the comparisons of the clinical presentation of anemia and depressive symptoms. The several covariates used in multivariate analysis are not only directly influenced by response variables but are also affected by other covariates which often hinders analysis. In this study, we did not take into the consideration the patients' BMI, psychosocial dimensions, including economic status, family support, gender differences, reproductive age and menopausal, and some other emotional stresses related to depressive symptoms. Previous study reported that there was a significant difference in the levels of serum ferritin concentrations between genders in a Japanese population [39]. In future research, we need to consider and remove the effect of confounding the patient background factors such as diseases associated with depression such as history of stroke and other neurological conditions, bed bound patients, and even social conditions such as monthly income, family support.

\section{Conclusion}

In summary, we found higher serum ferritin levels were associated with the presence of depressive symptoms among patients undergoing HD. Because iron metabolism in HD patients may be associated with depressive symptoms, we believe that careful iron management is necessary in HD treatment. our findings will be useful when considering adjustment of serum ferritin levels and TSAT for HD patients. In the clinical assessment of iron status, it must be kept in mind that inappropriate iron management of HD patients may be involved in the appearance of depressive symptoms.

\section{Disclosure Statement}

All authors declare no competing financial interests.

\section{Statement of Ethics}

All procedures performed in studies involving human participants were in accordance with the ethical standards of the institutional and national research committees at which the studies were conducted (IRB approval number NCT03096626) and with the 1964 Helsinki declaration and its later amendments or comparable ethical standards.

\section{Funding Sources}

Not applicable.

\section{Author Contributions}

YT designed the study. NS and YH analyzed and interpreted the data. TN, TT, and YMM drafted the article and revised it. All authors participated in drafting the article or revising it critically for important intellectual content. All authors read and approved the final manuscript.

\section{Acknowledgements}

The authors thank the staff of the dialysis centre at Tojinkai Hospital for their help with data collection.

\section{References}

[1] R Kimmel PL. Psychosocial factors in adult end-stage renal disease patients treated with hemodialysis: correlates and outcomes. Am J Kidney Dis 2000; 35: S132-S140J.

[2] Kimmel PL. Psychosocial factors in dialysis patients. Kidney Int 2001; 59: 1599-1613.

[3] Lopes AA, Bragg J, Young E, et al. Depression as a predictor of mortality and hospitalization among hemodialysis patients in the United States and Europe. Kidney Int 2002; 62: 199-207.

[4] Kimmel PL, Weihs K, Peterson RA. Survival in hemodialysis patients: the role of depression. J Am Soc Nephrol 1993; 4: 1227.

[5] Beard JL, Connor JR, Jones BC. Iron in the brain. Nutr Rev 1993; 51: 157-170.

[6] Roncagliolo M, Garrido M, Walter T, Peirano P, Lozoff B. Evidence of altered central nervous system development in infants with iron deficiency anaemia at 6 mo: delayed maturation of auditory brainstem responses. Am J Clin Nutr 1998; 68: 683-690.

[7] Tracey A. Rouault. Iron metabolism in the CNS: implications for neurodegenerative diseases. Nature reviews. Neuroscience, 2013; 14 (8): 551-564.

[8] Vahdat Shariatpanaahi M, Vahdat Shariatpanaahi Z, Moshtaaghi M, Shahbaazi SH, Abadi A. The relationship between depression and serum ferritin level. Eur J Clin Nutr 2007; 61: 532-535.

[9] Baune BT, Eckardstein AV, Berger K. Lack of association between iron metabolism and depressive mood in an elderly general population. Int Psychogeriatr 2006; 18: 337-444.

[10] Hunt JR, Penland JG. Iron status and depression in premenopausal women: an MMPI study. Minnesota Multiphasic Personality Inventory. Behav Med 1999; 25: 62-68.

[11] Baune BT, Neuhauser H, Ellert U, Berger K. The roles of inflammatory workers ferritin, transferrin and fibrinogen in the relationship between major depression and cardiovascular disorders - The German Health Interview and Examination Survey. Acta Psychiatr Scand 2010; 121: 135-142.

[12] Yamada K, Furuya R, Takita T, et al. Simplified nutritional screening tools for patients on maintenance hemodialysis. Am J Clin Nutr 2008; 87: 106-113.

[13] Matsuzawa Y, Tokunaga K, Kotani K, Keno Y, Kobayashi T, Tarui S. Simple estimation of ideal body weight from body mass index with the lowest morbidity. Diabetes Res Clin Pract 1990; 10 Suppl 1: S159-S164. 
[14] Examination Committee of Criteria for 'Obesity Disease' in Japan; Japan Society for the Study of Obesity. New criteria for 'obesity disease' in Japan. Circ J 2002; 66: 987-992.

[15] Beck AT, Steer RA, Ball R, Ranieri W. Comparison of Beck Depression Inventories -IA and -II in psychiatric outpatients. $J$ Pers Assess 1996; 67: 588-597.

[16] Wuerth D, Finkelstein SH, Ciarcia J, Peterson R, Kliger AS, Finkelstein FO. Identification and treatment of depression in a cohort of patients maintained on chronic peritoneal dialysis. Am J Kidney Dis 2001; 37: 1011-1017.

[17] Kimmel PL, Peterson RA, Weihs KL, et al. Multiple measurements of depression predict mortality in a longitudinal study of chronic hemodialysis outpatients. Kidney Int 2000; 57: 2093-2098.

[18] Beck AT, Steer RA, Brown GK. Manual for the Beck Depression Inventory-II. Psychological Corporation, San Antonio, TX: 1996.

[19] Simic Ogrizovic S, Jovanovic D, Dopsaj V, et al. Could depression be a new branch of MIA syndrome? Clin Nephrol 2009; 71: 164-172.

[20] Tsubakihara Y, Nishi S, Akiba T, et al. 2008 Japanese Society for Dialysis Therapy: guidelines for renal anaemia in chronic kidney disease. Ther Apher Dial 2010; 14: 240-275.

[21] Klipstein-Grobusch K, Koster JF, Grobbee DE, et al. Serum ferritin and risk of myocardial infarction in the elderly: the Rotterdam Study. Am J Clin Nutr 1999; 69: 1231-1236.

[22] Mezzano D, Pais EO, Aranda E, et al. Inflammation, not hyperhomocysteinemia, is related to oxidative stress and hemostatic and endothelial dysfunction in uremia. Kidney Int 2001; 60: 1844-1850.

[23] Feldman HI, Santanna J, Guo W, et al. Iron administration and clinical outcomes in hemodialysis patients. $J$ Am Soc Nephrol 2002; 13: 734-744.

[24] Kimmel PL, Phillips TM, Simmens SJ, et al. Immunologic function and survival in hemodialysis patients. Kidney Int 1998; 54: $236-244$.

[25] Beamish MR, Davies AG, Eakins JD, Jacobs A, Trevett D. The measurement of reticuloendothelial iron release using iron-dextran. Br J Haematol 1971; 21: 617-622.

[26] Macdougall IC, Hutton RD, Cavill I, Coles GA, Williams JD. Poor response to treatment of renal anaemia with erythropoietin corrected by iron given intravenously. $B M J$ 1989; 299: 157-158.

[27] Stadler N, Lindner RA, Davies MJ. Direct detection and quantification of transition metal ions in human atherosclerotic plaques: evidence for the presence of elevated levels of iron and copper. Arterioscler Thromb Vasc Biol 2004; 24: 949-954. Epub 2004 Mar 4.

[28] Drüeke T, Witko-Sarsat V, Massy Z, et al. Iron therapy, advanced oxidation protein products, and carotid artery intima-media thickness in end-stage renal disease. Circulation 2002; 106: 2212-2217.

[29] Sipe JC, Lee P, Beutler E. Brain iron metabolism and neurodegenerative disorders. Dev Neurosci 2002; 24: 188-196.

[30] Whitnall M, Richardson DR. Iron: a new target for pharmacological intervention in neurodegenerative diseases. Semin Pediatr Neurol 2006; 13: 186-197.

[31] Madsen E, Gitlin JD. Copper and iron disorders of the brain. Annu Rev Neurosci 2007; 30: 317-337.

[32] Sian-Hülsmann J, Mandel S, Youdim MB, Riederer P. The relevance of iron in the pathogenesis of Parkinson's disease. $J$ Neurochem 2011; 118: 939-957.

[33] Smith DG, Cappai R, Barnham KJ. The redox chemistry of the Alzheimer's disease amyloid beta peptide. Biochim Biophys Acta 2007; 1768: 1976-1990.

[34] Raven EP, Lu PH, Tishler TA, Heydari P, Bartzokis G. Increased iron levels and decreased tissue integrity in hippocampus of Alzheimer's disease detected in vivo with magnetic resonance imaging. $J$ Alzheimers Dis 2013; 37: 127136.

[35] Basu S, Mohan ML, Luo X, Kundu B, Kong Q, Singh N. Modulation of proteinase K-resistant prion protein in cells and infectious brain homogenate by redox iron: implications for prion replication and disease pathogenesis. Mol Biol Cell 2007; 18: 3302-3312. Epub 2007 Jun 13.

[36] Kalender B, Ozdemir AC, Koroglu G. Association of depression with markers of nutrition and inflammation in chronic kidney disease and end-stage renal disease. Nephron Clin Pract 2006; 102: c115-121.

[37] Chilcot J, Friedli K, Guirguis A, Wellsted D, Farrington K, Davenport A. C reactive protein and depressive symptoms in haemodialysis patients: A questionable association. Hemodial Int 2016 Sep 27. doi: 10.1111/hdi.12500. [Epub ahead of print].

[38] Friend R, Hatchett L, Wadhwa NK, Suh H. Serum albumin and depression in end-stage renal disease. Adv Perit Dial 1997; 13: 155-157.

[39] Yi S, Nanri A, Poudel-Tan dukar K, Nonaka D, Matsushita Y, Hori A, et al. Association between serum ferritin concentrations and depressive symptoms in Japanese municipal employees. Psychiatry research. 2011; 189 (3): 368-721. 